\title{
Heimtiere und Gesundheit
}

Robert Tanner,

Dennis C. Turner
Man hört und liest in populären Medien immer wieder, dass Heimtiere wie Hunde, Katzen, Kaninchen, ja sogar manche Bauernhoftiere, die Gesundheit und das emotionale Wohlbefinden von Menschen unterschiedlicher Altersklassen positiv beeinflussen können. Tiere, die gut sozialisiert wurden und artgerecht aufgewachsen sind, können therapeutisch und pädagogisch eingesetzt werden. Tiergestützte Interventionen helfen Menschen, die unter bestimmten psychischen und/oder physischen Krankheiten leiden, zu mehr Lebensqualität.

\section{Diese Qualifikation befähigt die Absolventen, in der Ausübung ihres Berufes professionell Tiere einzubeziehen.}

1 Julius H, Beetz A, Kotrschal K, Turner D, Uvnäs-Moberg K. Attachment to Pets.

An integrative view of human-animal relationships with implications for therapeutic practice. (In Vorbereitung). Göttingen: Hogrefe; 2013.

Korrespondenz:

Dr. med. Robert Tanner-Frick Facharzt für Kinder- und Jugendpsychiatrie und Psychotherapie Gemeinschaftspraxis Altamira Iddastrasse 51 CH-9008 St. Gallen Tel. 0712453825 Fax 0712453826 info[at]tiergestuetztepsychotherapie.ch
Über die letzten drei Jahrzehnte sind mehrere Forschungsberichte darüber in anerkannten medizinischen, pädagogischen und psychologischen Fachzeitschriften, wie z. B. American Journal of Cardiology, Nursing Research, Medical Journal of Australia, Journal of the Royal Society of Medicine, JAMA, Journal of Nervous and Mental Disease, British Journal of Psychology, veröffentlicht worden, die diese Annahmen wissenschaftlich unterstützen. Diesen Monat erscheint ein neues Buch (zuerst auf Englisch, gegen Ende 2013 auf Deutsch): «Attachment to Pets. An integrative view of human-animal relationships with implications for therapeutic practice» [1], das die evolutionären, biophysiologischen und psychologischen Prozesse integrativ als Erklärung der positiven Wirkfaktoren der Mensch-Tier-Beziehungen auf unser Wohlbefinden, respektive unsere Gesundheit zusammenfassend darstellt.

Tiergestützte Interventionen, der Überbegriff für tiergestützte Therapie (TGT), tiergestützte (Sonder-) Pädagogik und tiergestützte Fördermassnahmen, geniessen immer breiteres Interesse und Anerkennung in Fachkreisen (s. www.aat-isaat.org und www.gtta.ch) sowie in der breiten Öffentlichkeit. Es ist wichtig, dass die verschiedenen Fachstellen, inkl. Ärzteschaft, Spi- täler und Kliniken, die Möglichkeiten und Grenzen der TGT, wie auch die Unterschiede zwischen tiergestützter Therapie (TGT) und tiergestützten Fördermassnahmen (TGF) kennen, und die Qualifikationen der Anbieter solcher Dienste beurteilen können.

Die ISAAT, gegründet im November 2006 (International Society for Animal-Assisted Therapy) hat daher strenge Weiterbildungskriterien für Therapeutinnen und Therapeuten sowie Pädagogen, die Tiere in ihrem Berufsfeld unterstützend einbeziehen, erarbeitet. Es gibt nur eine Institution in der Schweiz, das eduQua-zertifizierte I.E.T. (Institut für Ethologie und Tierpsychologie (www.turner-iet.ch/de/ausbil dung.php), das in Zusammenarbeit mit der Psychotherapeutischen Praxis Altamira (Dres. med. R. Tanner-Frick und phil. E. Frick Tanner) in St. Gallen die ISAAT Anerkennung erhalten hat und seit 1999 berufsbegleitende Ausbildungskurse in Zürich anbietet. Diese Qualifikationen befähigen die Absolventinnen und Absolventen in der Ausübung ihres Berufes, professionell Tiere einbeziehen zu können.

Die I.E.T.-Absolvent(inn)en, die eigene therapeutische oder pädagogische Angebote auf privater Basis oder in Institutionen ausführen, sind zusammengeschlossen im schweizerischen Berufsverband, Gesellschaft für Tiergestützte Therapie und Aktivitäten (GTTA).

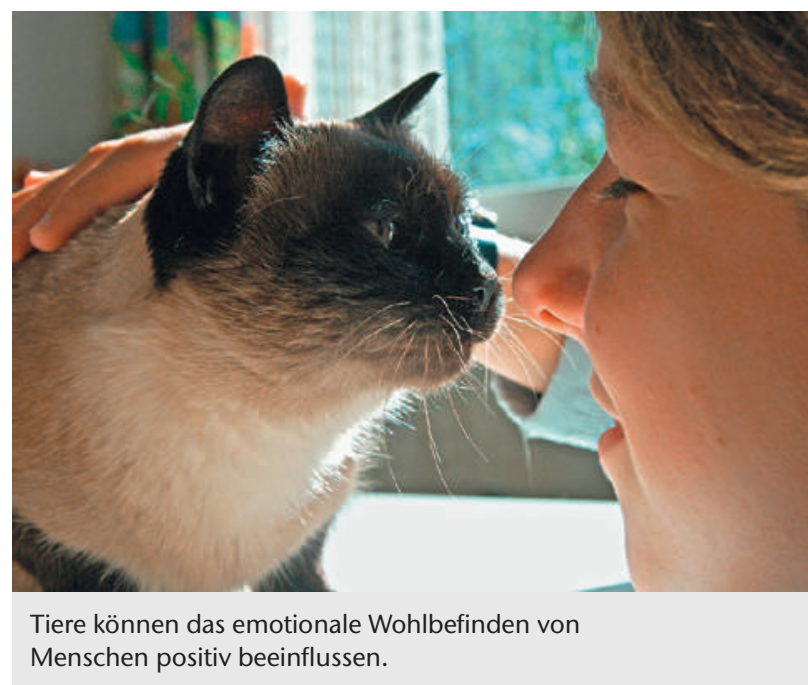

